\title{
Impacts de la Dynamique des Paysages Agraires sur les Activités Agricoles dans la Commune d'adéane en Basse-Casamance (Sénégal)
}

\author{
Alexandre Badiane, \\ Tidiane Sané, \\ Mamadou Thior,
}

Laboratoire de Géomatique et d'Environnement (LGE), Université Assane SECK de Ziguinchor, Ziguinchor, Sénégal, Laboratoire Mixte International «Patrimoines et Territoires de l'Eau» (LMI PATEO), Institut de Recherche pour le Développement (IRD)

Unité Mixte de Recherche 208 PALOC, MNHN-IRD, Paris

Doi:10.19044/esj.2019.v15n21p489 URL:http://dx.doi.org/10.19044/esj.2019.v15n21p489

\section{Resume:}

La présente étude vise à comprendre la dynamique des paysages agraires et ses impacts sur les activités agricoles dans la commune d'Adéane dans un contexte de dégradation des conditions agro-pédologiques. La cartographie diachronique d'occupation des sols de 1968, 2004 et 2016, réalisée avec Arc Gis (10.2), a permis de quantifier l'évolution spatiotemporelle des paysages agraires. Les mesures in situ effectuées dans les rizières, à l'aide du combo Hanna 87130 (pH et conductivité électrique) et du réfractomètre, mettent en évidence les phénomènes de salinisation (avec un maximum de $25 \%$ o dans les rizières d'Adéane), et de l'acidification ( $\mathrm{pH}$ de 4 à d'Agnack-petit), à l'origine de la dégradation des conditions agropédologiques. La principale conséquence est le recul des rizières exploitées (45,45\% pour Adéane et 56,65\% pour Agnack-peti entre 1968-2016) et de la forêt claire (100\% pour Adéane et Agnack-petit entre 1968-2016) au profit de l'arboriculture d'anacardiers qui a progressé de 228,46 \% à Adéane et de $519,28 \%$ à Agnack-petit. Face à un développement rapide de l'arboriculture d'anacardier dans la commune d'Adéane et au recul des activités agricoles (riziculture et culture de plateau), il demeure important de s'interroger sur la durabilité des systèmes agricoles dans cette Commune dans un contexte de transition agroécologique.

Mots clés : Arboriculture d'anacardiers, Occupation des sols, Dynamique, Paysages agraires, Riziculture. 


\title{
Impacts of the Dynamics of Aging Landscapes on Agricultural Activities in the Commune of Adéane in Lower Casamance (Senegal)
}

\author{
Alexandre Badiane, \\ Tidiane Sané, \\ Mamadou Thior,
}

Laboratoire de Géomatique et d'Environnement (LGE), Université Assane SECK de Ziguinchor, Ziguinchor, Sénégal, Laboratoire Mixte International «Patrimoines et Territoires de l'Eau» (LMI PATEO), Institut de Recherche pour le Développement (IRD)

Unité Mixte de Recherche 208 PALOC, MNHN-IRD, Paris

\begin{abstract}
This study aims at understanding the dynamics of agrarian landscapes and their impacts on agricultural activities in the town of Adéane in a context of deteriorating agro-pedological conditions. The diachronic cartography of land use of 1968, 2004 and 2016, carried out with Arc Gis (10.2), made it possible to quantify the spatio-temporal evolution of agrarian landscapes. In situ measurements made in the rice fields, using the Hanna 87130 combo (pH and electrical conductivity) and the refractometer, highlight salinisation phenomena (with a maximum of $25 \% \mathrm{o}$ in the Adéane rice paddy fields), and acidification ( $\mathrm{pH} 4$ to Agnack-small), causing degradation of agro-pedological conditions. The main consequence is the decline of exploited rice fields (45.45\% for Adéane and 56.65\% for Agnack-peti between 1968-2016) and light forest (100\% for Adéane and Agnack-petit between 1968-2016) benefit of the arboriculture of cashew trees which rose by $228.46 \%$ in Adéane and $519.28 \%$ in Agnack-petit. In view of the rapid development of cashew growing in the commune of Adéane and the decline of agricultural activities (rice growing and plateau farming), it remains important to question the sustainability of agricultural systems in this Commune in a context of agroecological transition.
\end{abstract}

Keywords: Arboriculture of cashew trees, Land cover, Dynamics, Agrarian landscapes, Rice growing 


\section{Introduction}

La péjoration climatique notée durant les décennies 1970 à 1990, en Afrique de l'Ouest, a eu des répercussions sur les ressources naturelles et les activités socioéconomiques du monde rural. Au Sénégal, plus particulièrement en Basse Casamance, la riziculture qui occupe une place prépondérante dans l'alimentation des familles, subi les contres-coup de cette péjoration climatique. Les rizières de bas-fonds ont été alors progressivement contaminées par les eaux de surface sursalées (Cormier-Salem, 1992; Marius \& Lucas, 1982) entraînant parfois leur dégradation et leur abandon. Malgré les nombreux efforts d'aménagement consentis dans le secteur de la riziculture construction de barrages, de digues anti-sel et d'ouvrages évacuateurs (Badiane, 2016b; Diouf, 2013; Sané, 2017) et le retour à la normale des précipitations observé ces dernières décennies (Descroix et al., 2016), la superficie des rizières exploitées ne cessent de reculer et la production. Face à cette situation, certains paysans de la Basse-Casamance s'orientent de plus en plus vers d'autres activités telles que le tourisme et de la pêche dans les communes de Diembering et Kafoutine (Sané, 2017; Thior et al., 2019a), du maraîchage et de la plantation d'arbres fruitiers (Badiane, 2016a).

Dans la commune d'Adéane, la reconversion professionnelle des paysans vers l'arboriculture d'anacardiers sur les zones de culture de plateau, est très marquée. Cette activité occupe aujourd'hui une place de choix dans l'économie locale. Elle est la principale source de revenus des populations et menace de près la riziculture et les autres cultures céréalières (mil, mais, sorgho...) et arachidières (Badiane, 2016a). La production actuelle de céréale est loin de couvrir les besoins alimentaires des paysans. Grâce aux revenus tirés de la production d'anacarde, les paysans font recours aux céréales importées pour combler le déficit alimentaire (Badiane, 2016a ; Ndiaye et al., 2017).

Le présent article met l'accent, à partir des outils de la géomatique, sur l'analyse spatio-temporelle de la dynamique des paysages agraires de la commune d'Adéane entre 1968 et 2016. Il met en évidence, d'une manière générale, l'état de dégradation avancé des rizières de bas-fonds et le recul des champs de cultures et de la forêt claire au profit de l'arboriculture d'anacardiers. Il tente ensuite d'expliquer les facteurs à l'origine de cette dynamique et analyse les conséquences de cette dynamique sur l'agriculture de la commune d'Adéane.

\section{Matériel et Méthodes}

\subsection{Présentation de la zone d'étude}

Située à $12^{\circ} 37^{\prime} 48^{\prime \prime}$ nord et $16^{\circ} 01^{\prime} 01^{\prime \prime}$ ouest, à l'extrême est du département de Ziguinchor (au sud du Sénégal), la commune d'Adéane fait partie de l'arrondissement de Niaguis. Elle est limitée à l'est par 
l'arrondissement de Diattacounda, à l'ouest par la commune de Niaguis, au nord par le fleuve Casamance et au sud par la commune de Boutoupa Camaracounda (figure 1).

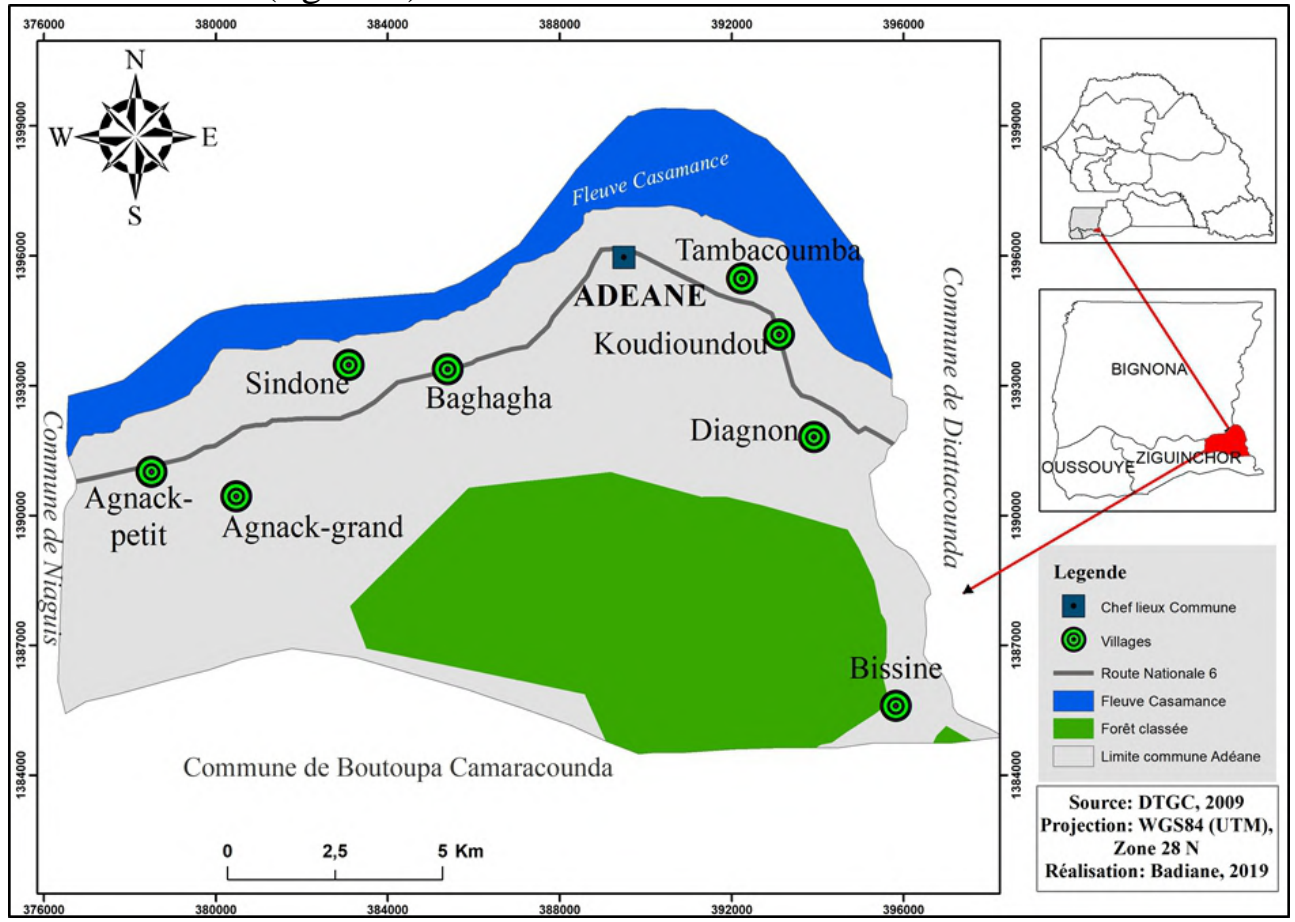

Figure 1: Carte de localisation de la commune d'Adéane

La commune d'Adéane s'étend sur une superficie de $192 \mathrm{~km}^{2}$, dont $42 \mathrm{~km}^{2}$ occupés par la forêt classée de Bissine (Communauté Rurale d'Adéane, 2008). Sa population s'élève à 17580 habitants (ANSD, 2017) répartis sur neuf villages. Cette population est caractérisée par une importante diversité ethnique avec cependant une prédominance des Mandingues et des Diolas avec respectivement 30 et $20 \%$ (Badiane, 2016a). L'agriculture, notamment l'arboriculture d'anacardiers, reste aujourd'hui la principale activité des populations de cette commune à laquelle s'ajoute la pêche généralement pratiquée par les populations non autochtones venues du nord du Sénégal et de la sous-région (Mali).

\subsection{Méthodes}

La démarche méthodologique adoptée dans le cadre de ce travail est basée sur le croisement des données cartographiques, physico-chimiques et des données d'enquête de terrain. L'intérêt d'adopter une telle démarche réside dans le sens où les données cartographiques ont permis de quantifier l'évolution des classes d'occupation des sols au niveau des bas-fonds et plateaux. Les données physico-chimiques ont permis de comprendre les 
facteurs physiques et chimiques responsables de la dégradation des rizières de bas-fonds. Enfin, les données d'enquête de terrain ont permis de comprendre la perception des populations sur les facteurs de la dynamique des paysages agraires et les conséquences de cette dynamique sur les activités agricoles dans la commune d'Adéane.

Les données cartographiques sont obtenues à partir du téléchargement des images satellites Corona de 1968 et des images Google Earth de 2004 et 2016. Cette étape est suivie par l'observation et la prise de points GPS sur le terrain. Ces images ont fait l'objet de traitement dans ArcGIS (version 10.2), suivant quatre étapes : le mosaïquage, le géoréférencement, la numérisation des classes d'occupation des sols et la réalisation des cartes multi-dates d'occupation des sols de 1968, 2004 et 2016. Dans l'objectif de mieux comprendre la dynamique des paysages agraires de la Commune d'Adéane, la cartographie à l'échelle de deux terroirs villageois (Adéane et Agnack-Petit) a été réalisé. Plusieurs raisons fondent ce choix. D'une part, ces deux terroirs regroupent une bonne partie des terres agricoles de la Commune d'Adéane, qui sont également exploitées par les paysans des villages environnants. Par exemple, les rizières d'Agnack-Petit sont exploitées aussi par les paysans des villages de Sinedone et de Agnack grand. D'autre part, la dégradation des rizières est beaucoup plus ressentie dans ces deux terroirs du fait de la présence de cours d'eau salés. Par ailleurs, le phénomène le plus marquant au niveau du plateau est le défrichement de la forêt claire dans ces terroirs. Ce déboisement est lié à la conquête de nouvelles terres pour la plantation des anacardiers.

Il a été d'abord réalisé des cartes d'occupation des sols pour chacune des années retenues (1968, 2004 et 2016), puis les superficies des différentes classes d'occupation des sols ont été calculée. Enfin, il a été calculé les taux d'évolution entre 1968-2004, 2004-2016 et 1968-2016 pour chaque classe d'occupation des sols selon la formule suivante : ((Aa-Ad) /Ad) $* \mathbf{1 0 0}{ }^{1}$.

Les données physico-chimiques ont été obtenues à partir des mesures in situ de l'eau, effectuées dans les rizières exploitées et les rizières abandonnées de la commune d'Adéane. Le potentiel d'Hydrogène $(\mathrm{pH})$, la Conductivité Electrique $(\mathrm{CE})$ et la Réfractométrie sont les trois paramètres physico-chimiques mesurés et qui ont permis de déterminer le niveau de salinité et d'acidité des eaux des rizières.

Les données d'enquêtes (questionnaire) ont été collectées selon deux méthodes d'échantillonnage. Dans un premier temps, sur la base des données de l'ANSD, un échantillonnage aléatoire simple a été effectué. Il a permis d'interroger $15 \%$ des ménages de la Commune d'Adéane, soit 198 ménages sur 1318. Pour obtenir la taille de l'échantillon, la formule $\left(n=N^{*} 15 / 100\right)^{2}$

${ }^{1}$ (Aa) représente l'année d'arrivée et (Ad) l'année de départ.

2 (n) représente la taille de l'échantillon, qui est égale à 198 ménages ; $(\mathbf{N})$ représente la population cible ou le nombre total de ménages estimé à 1318 ménages. 
proposée par Diatta et Diouf (2013) a été utilisée. Par la suite, la méthode d'échantillonnage par quota, en multipliant le nombre de ménages de chaque village par un taux de $15 \%$, a été adopté pour déterminer l'échantillon dans chaque village.

Tableau I : Récapitulatif du nombre de ménages interrogés par village

\begin{tabular}{|c|c|c|c|}
\hline Villages & Ménages & $\begin{array}{c}\text { Nombre de ménages } \\
\text { interrogés }\end{array}$ & $\begin{array}{c}\text { Pourcentage des ménages } \\
\text { interrogés }(\%)\end{array}$ \\
\hline Adéane & 369 & 55 & 28 \\
\hline Agnack-petit & 230 & 35 & 17,4 \\
\hline Baghagha & 219 & 33 & 16,6 \\
\hline Sindone & 156 & 23 & 11,8 \\
\hline Tambacoumba & 113 & 17 & 8,6 \\
\hline Diagnon & 80 & 12 & 6,1 \\
\hline Gonoum & 59 & 9 & 4,5 \\
\hline Koundioundou & 53 & 8 & 4 \\
\hline Sibinck & 23 & 4 & 1,8 \\
\hline Agnack Grand & 16 & 2 & 1,2 \\
\hline Total & 1318 & 198 & 100 \\
\hline
\end{tabular}

Source des données : ANSD, 2002

Les enquêtes ménages sont complétées par les guides d'entretiens semi-directifs réalisés auprès du représentant du projet Natal MBAYE dans la commune d'Adéane, le Directeur de l'ANCAR ${ }^{3}$ et les responsables de suivi des digues et ouvrages construits par les projets/programmes (tableau II).

Tableau II : Liste des personnes ressources

\begin{tabular}{|l|l|}
\hline Personnes ressources & Responsabilités \\
\hline Ibrahima Badiane & Directeur régional de l'agence national de conseil agricole rural \\
\hline Sadia Sonko & $\begin{array}{l}\text { Responsable de suivi des digues et ouvrages construits à } \\
\text { Adéane, Producteur d'anacarde }\end{array}$ \\
\hline Kéba kombo & $\begin{array}{l}\text { Chef de village de Agnack-petit, responsable de suivi des } \\
\text { digues et ouvrages construits à Agnack-petit }\end{array}$ \\
\hline Phillippe Diédhiou & Relai communautaire, Producteur d'anarcarde \\
\hline Yancouba cissé & $\begin{array}{l}\text { Représentant du Projet Natal Mbaye dans la commune } \\
\text { d'Adéane }\end{array}$ \\
\hline Bassamba Diédhiou & Secrétaire Général de la chambre de commerce de Ziguinchor \\
\hline
\end{tabular}

Les informations obtenues de ces enquêtes et entretiens ont facilité l'analyse des résultats cartographiques et physico-chimiques.

${ }^{3}$ Agence Nationale de Conseil Agricole Rurale 


\section{Résultats}

\subsection{Dynamique des paysages agraires de la Commune d'Adéane}

L'étude de la dynamique des paysages agraires de la Commune d'Adéane révèle la complexité du milieu liée à la diversité des composantes de l'écosystème et des facteurs qui les font évoluer. Dans cette partie, l'accent a été mis sur la compréhension de la dynamique de l'occupation des sols au niveau du plateau et des bas-fonds. La figure 2 représente l'occupation des sols du terroir d'Adéane de 1968, 2004 et 2016.

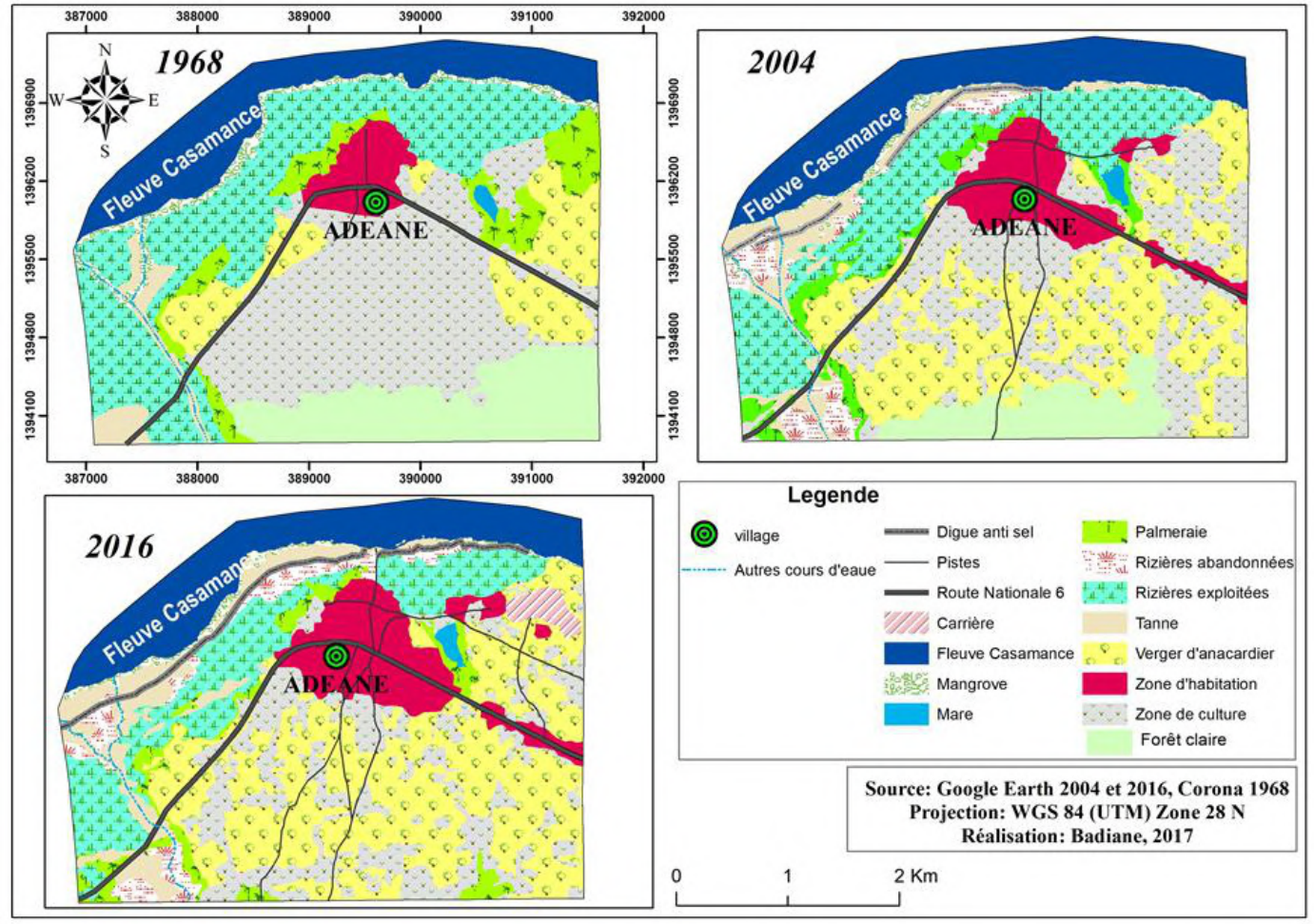

Figure 2: Carte d'occupation des sols du terroir d'Adéane de 1968, 2004 et 2016

Les résultats cartographiques, illustrés par la figure 2 montrent une dynamique relativement importante des classes d'occupation des sols des terroirs villageois d'Adéane entre 1968, 2004 et 2016. Cette dynamique est perceptible à travers l'évolution des différentes classes d'occupation des sols (rizières exploitées, rizières abandonnées, mangrove, forêt claire, zone de culture de plateau, verger d'anacardiers, zone d'habitation, tanne, palmeraie). Il en résulte, d'une part, que les rizières exploitées, les zones de culture de plateau, la forêt claire entre autres ont baissé entre 1968, 2004 et 2016 et d'autre part, les classes arboriculture d'anacardiers, rizières abandonnées ont progressé. Les statistiques (superficies en hectare et le taux d'évolution) des différentes classes d'occupation des sols sont représentées dans le tableau III. 
Tableau III : Évolution des classes d'occupation des sols du terroir d'Adéane entre 19682004 ; 2004-2016 et 1968-2016

\begin{tabular}{|l|c|c|c|c|c|c|}
\hline \multirow{2}{*}{ Classes } & \multicolumn{3}{|c|}{ Annerficies en hectare (ha) } & \multicolumn{2}{c|}{$\begin{array}{c}\text { Taux d'évolution en pourcentage (\%) } \\
\text { (Progression/régression) }\end{array}$} \\
\cline { 2 - 8 } & $\mathbf{1 9 6 8}$ & $\mathbf{2 0 0 4}$ & $\mathbf{2 0 1 6}$ & $\mathbf{1 9 6 8 - 2 0 0 4}$ & $\mathbf{2 0 0 4 - 2 0 1 6}$ & $\mathbf{1 9 6 8 - 2 0 1 6}$ \\
\hline Carrière & 0 & 0 & 16,87 & - & 100,00 & 100,00 \\
\hline Fleuve Casamance & 188,93 & 190,42 & 197,14 & 0,78 & 3,52 & 4,34 \\
\hline Forêt claire & 179,22 & 76,2 & 0 & $-57,48$ & $-100,00$ & $-100,00$ \\
\hline Mangrove & 41,84 & 12,45 & 20,14 & $-70,24$ & 61,76 & $-51,86$ \\
\hline Mares & 3,84 & 4,85 & 5,3 & 26,30 & 9,27 & 38,02 \\
\hline Palmeraies & 94,91 & 49,72 & 60,75 & $-47,61$ & 22,18 & $-35,99$ \\
\hline Rizières abandonnées & 0 & 49,39 & 61,95 & 100,00 & 25,43 & 100,00 \\
\hline Rizières exploitées & 346,01 & 210,18 & 188,72 & $-39,25$ & $-10,21$ & $-45,45$ \\
\hline Tannes & 36,2 & 70,45 & 100,92 & 94,61 & 43,25 & 178,78 \\
\hline Vergers d'anacardiers & 144,37 & 351,4 & 474,21 & 143,40 & 34,94 & 228,46 \\
\hline Zones de culture & 425,34 & 403,58 & 250,41 & $-5,11$ & $-37,95$ & $-41,12$ \\
\hline Zones d'habitation & 54,54 & 96,56 & 138,79 & 77,04 & 43,73 & 154,47 \\
\hline Totaux & $\mathbf{1 5 1 5 , 2}$ & $\mathbf{1 5 1 5 , 2}$ & $\mathbf{1 5 1 5 , 2}$ & - & - & - \\
\hline
\end{tabular}

Source : Image Corona 1968 et images Google Earth 2004 et 2016

Les données du tableau III montrent de manière claire les superficies et les taux d'évolution des différentes classes d'occupation des sols, notamment en termes de régression et de progression. L'analyse de ce tableau, laisse apparaître une importante régression des superficies de rizières exploitées entre 1968, 2004 et 2016 au niveau des zones inondées, laissant la place aux rizières abandonnées et à d'importantes surfaces de tanne. Ainsi, les rizières exploitées ont connu un important taux de régression allant de 39,25 $\%$ entre $1968-2004,10,21 \%$ entre $2004-2016$ et $45,45 \%$ entre $1968-2016$ alors que les rizières abandonnées connaissent un taux de progression de $100 \%$ entre 1968-2004 et 1968-2016 et 25,43\% entre 2004-2016 (tableau III). Par ailleurs, la mangrove a connu une importante régression entre 1968 et 2004 avec un taux de 70,24 \%. Cependant, on note un retour des écosystèmes de mangrove dans les terroirs d'Adéane entre 2004 et 2016 avec un taux de progression de $61,76 \%$. Ce retour de la mangrove est surtout lié à l'intervention de l'ONG OCEANIUM dans son programme de restauration des écosystèmes de mangrove en Basse Casamance.

$\mathrm{Au}$ niveau des zones exondées, le fait le plus marquant concerne surtout l'importante diminution des zones de cultures de plateau et des palmeraies, ainsi que la disparition de la forêt claire au profit des vergers d'anacardiers. La situation de la forêt claire est alarmante, car elle a existé entre 1968 et 2004 avec un taux de régression de 57,48 \% (tableau III), puis elle a complètement disparu entre 2004 et 2016 laissant la place aux vergers d'anacardiers et aux zones de cultures de plateau (figures 2). Quant à la classe 
"vergers d'anacardiers", elle a connu une très importante progression $(143,40$ $\%$ entre 1968-2004 à 228,46 \% entre 1968-2016). Ces mêmes tendances d'évolution sont notées dans les paysages agraires du terroir d'Agnack-petit (figure 3).

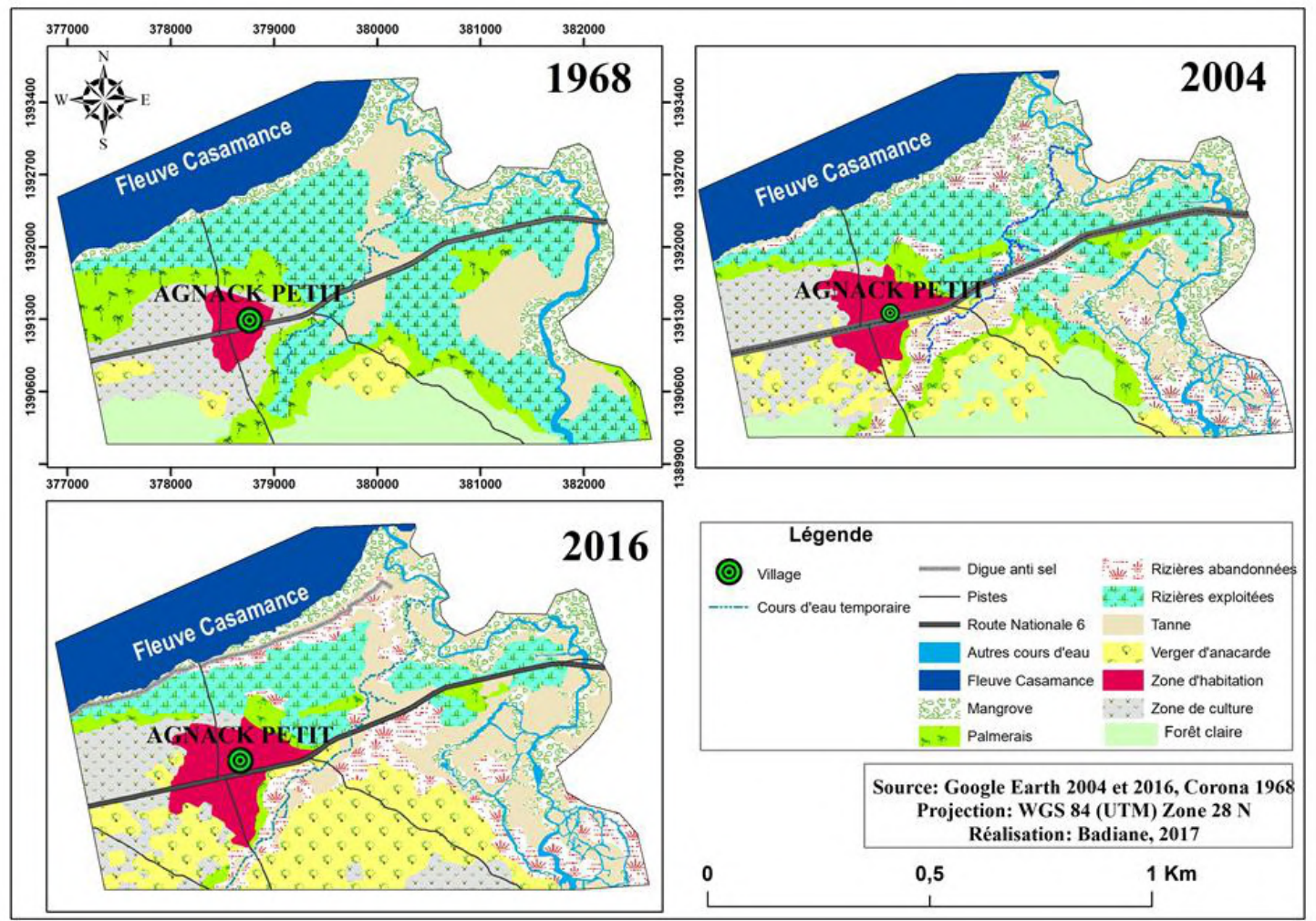

Figure 3 : Carte d'occupation des sols du terroir villageois d'Agnack-Petit de 1968, 2004 et 2016

À l'image du terroir d'Adéane, les résultats cartographiques du terroir de Agnack-petit révèle une évolution quasi-similaire des classes d'occupation des sols entre 1968, 2004 et 2016. Les rizières exploitées ont progressivement diminué et parallèlement, l'arboriculture d'anacardier évolue de manière rapide sur les zones de culture de plateau et sur la forêt claire (figure 3). Le tableau IV représente les statistiques des superficies et des taux d'évolution de chaque classe d'occupation des sols de 1968, 2004 et 2016. 
Tableau IV : Évolution des classes d'occupation des sols du terroir d'Agnack-Petit entre 1968-2004 ; 2004-2016 et 1968-2016

\begin{tabular}{|l|c|c|c|c|c|c|}
\hline \multirow{2}{*}{ Classes } & \multicolumn{3}{|c|}{ Années } & \multicolumn{2}{|c|}{ Superficies en hectare (ha) } & \multicolumn{2}{c|}{$\begin{array}{r}\text { Taux d'évolution en pourcentage (\%) } \\
\text { (Progression et régression) }\end{array}$} \\
\cline { 2 - 8 } & $\mathbf{1 9 6 8}$ & $\mathbf{2 0 0 4}$ & $\mathbf{2 0 1 6}$ & $\mathbf{1 9 6 8 - 2 0 0 4}$ & $\mathbf{2 0 0 4 - 2 0 1 6}$ & $\mathbf{1 9 6 8 - 2 0 1 6}$ \\
\hline Autres cours d'eau & 25,34 & 30,6 & 40,27 & 20,75 & 31,60 & 58,91 \\
\hline Fleuve Casamance & 206,22 & 210,19 & 220,85 & 1,95 & 5,07 & 7,09 \\
\hline Forêt claire & 171,61 & 78,2 & 0 & $-54,43$ & $-100,00$ & $-100,00$ \\
\hline Mangrove & 173,66 & 85,77 & 152,7 & $-50,61$ & 78,03 & $-12,06$ \\
\hline Palmeraies & 167,35 & 68,77 & 47,56 & $-58,90$ & $-30,84$ & $-71,58$ \\
\hline Rizières abandonnées & 0 & 101,08 & 164,04 & 100,00 & 62,28 & 100,00 \\
\hline Rizières exploitées & 410,53 & 330,88 & 177,97 & $-19,40$ & $-46,21$ & $-56,64$ \\
\hline Tannes & 169,68 & 282,73 & 235,74 & 68,39 & $-16,62$ & 38,93 \\
\hline Vergers d'anacardier & 43,6 & 90,8 & 270,01 & 108,25 & 197,35 & 519,28 \\
\hline Zones de culture & 130,81 & 187,84 & 135,1 & 43,59 & $-28,07$ & 3,27 \\
\hline Zones d'habitation & 32,85 & 64,79 & 87,41 & 97,22 & 34,91 & 166,08 \\
\hline Totaux & $\mathbf{1 5 3 1 , 6 5}$ & $\mathbf{1 5 3 1 , 6 5}$ & $\mathbf{1 5 3 1 , 6 5}$ & - & - & - \\
\hline
\end{tabular}

Source : Image Corona 1968 et images Google Earth 2004 et 2016

D'une manière générale, l'analyse du tableau IV montre, d'une part, une régression relativement importante des classes rizière exploitée, forêt claire et palmeraie, et d'autre part, une progression remarquable des classes rizières abandonnées, vergers d'anacardier, zones d'habitation entre autres. Les rizières exploitées ont connu un taux de régression de 19,40\% entre 19682004, de 46,21\% entre 2004-2016 et de 56,64\% entre 1968-2016 (tableau IV). Par conséquent, les rizières abandonnées ont progressé au fil du temps avec un taux de $100 \%$ entre 1968-2004 et 1968-2016 et 62,28 \% entre 20042016 (tableau IV).

Par ailleurs, la progression des vergers d'anacardiers au niveau du plateau est remarquable avec un taux de 108,25 \% entre 1968-2004, 197,35\% entre 2004-2016 et 519,28 \% entre 1968-2016. Parallèlement, la forêt claire connait une importante régression (100\% entre 1968-2004 et 1968-2016, 54,43\% entre 2004-2016) au profit de l'arboriculture d'anacardier. Quant aux classes mangrove, tanne et zone de culture de plateau, elles ont connu une évolution irrégulière. La mangrove a connu un taux de régression de 50,61\% entre 1968-2004, puis elle a progressé de 78,03 \% entre 2004-2016, en raison des interventions de reboisement effectuées par l'OCEANIAM en collaboration avec la population locale.

Ainsi, on peut noter que les deux terroirs villageois (Adéane et Agnack-petit) présentent une quasi-similarité du point de vue de l'évolution des classes d'occupation des sols. Au niveau des bas-fonds, le constat général est l'importante baisse des superficies de rizières exploitées, alors qu'au niveau du plateau le phénomène observé est l'extension spatiale des vergers 
d'anacardiers et des zones d'habitation sur d'anciens champs de cultures et les zones de forêt claire. Cette situation s'explique par un ensemble de facteurs.

\subsection{Principaux facteurs de la dynamique des paysages agraires}

L'analyse des informations issues des enquêtes réalisées auprès des ménages révèle la diversité des facteurs à l'origine de la dynamique des paysages agraires des terroirs de la commune d'Adéane. La figure 6 représente les principaux facteurs naturels de cette dynamique mis en avant par les ménages interrogés.

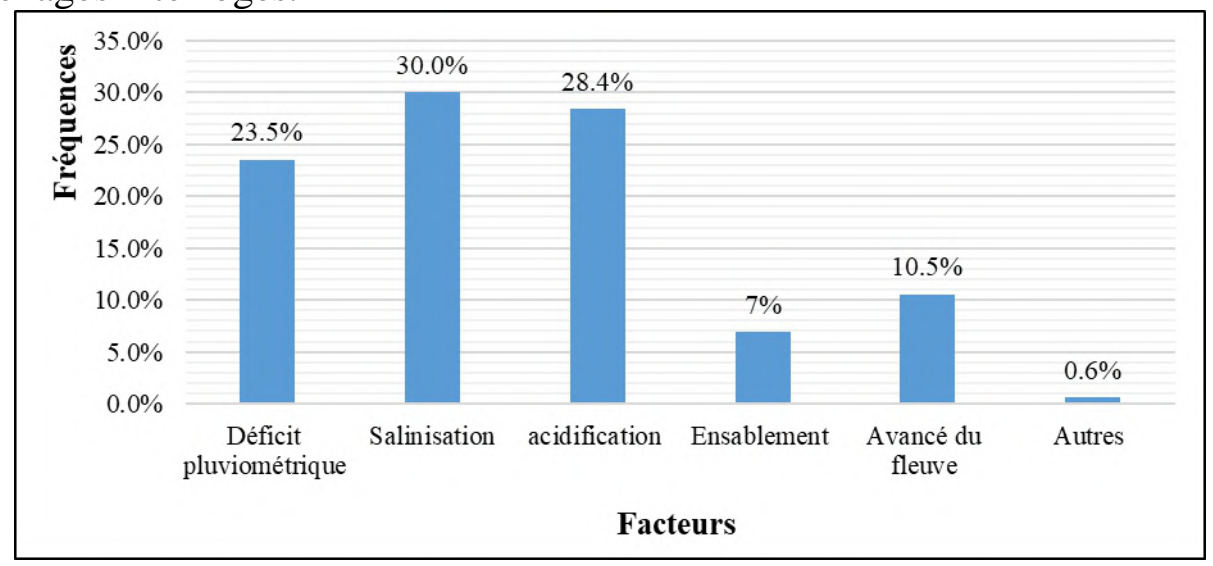

Figure 4 : Facteurs naturels de la dynamique du paysage agraire de la commune d'Adéane selon la population

La figure 4 montre que les phénomènes de salinisation, d'acidification et le déficit pluviométrique sont respectivement perçus par la population comme étant les principaux facteurs à l'origine de la dynamique des paysages au niveau des bas-fonds. En effet, la salinisation et l'acidification constituent les principaux processus qui conduisent à la dégradation chimique des sols (Centre de Suivi Ecologique, 2010). Une bonne partie des terres rizicoles de la commune d'Adéane sont sous l'emprise du sel et de l'acide. Le tableau 4 représente les données physico-chimiques issues des mesures in situ effectuées dans les rizières abandonnées et exploitées d'Adéane et d'AgnackPetit.

Tableau $\mathbf{V}$ : Résultats des paramètres physico-chimiques mesurés dans les eaux des rizières des terroirs d'Adéane et d'Agnack

\begin{tabular}{|l|l|c|c|c|c|c|}
\hline Villages & Entités & \multicolumn{2}{|c|}{ Coordonnées GPS } & Salinité \%o & CE $\boldsymbol{\mu s} / \mathbf{c m}$ & pH \\
\hline \multirow{2}{*}{ Adéane } & Latitude & Longitude & & & \\
\hline \multirow{2}{*}{$\begin{array}{l}\text { Agnack- } \\
\text { Petit }\end{array}$} & Rizières exploitées & 389380 & 1396773 & 5 & 800 & 7,8 \\
\cline { 2 - 7 } & Rizières abandonnées exploitées & 389445 & 1396848 & 25 & 20000 & 8,6 \\
\cline { 2 - 7 } & Rizières abandonnées & 380508 & 1391930 & 3 & 600 & 6,4 \\
\hline
\end{tabular}

Source : Badiane, 2016a et mesures in situ effectuées le 29 novembre 2016 
D'une manière générale, l'observation des résultats du tableau 4 montre que les rizières du terroir d'Adéane sont plus affectées par le phénomène de la salinisation que celles d'Agnack-Petit. Le taux de salinité obtenu dans ces rizières est de $25 \%$ avec une conductivité électrique excessive de $20000 \mu \mathrm{s} / \mathrm{cm}$. Par ailleurs, les rizières du terroir d'Agnack-Petit sont plus affectées par l'acidification, avec un $\mathrm{pH}$ de 4 au niveau des rizières abandonnées et 6,4 au niveau des rizières exploitées (tableau 4). Il faut également noter que les rizières abandonnées d'Agnack-petit concentrent un taux de salinité relativement important $(1700 \mu \mathrm{s} / \mathrm{cm}$ au niveau des rizières abandonnées et $600 \mu \mathrm{s} / \mathrm{cm}$ pour les rizières exploitées). Cette situation reste inquiétante du fait que les seules rizières exploitées dans ces deux terroirs sont actuellement sous la menace sans cesse de la salinité et de l'acidité. La forte salinité constatée, surtout dans les rizières d'Adéane, s'explique par leur proximité avec le fleuve Casamance qui favorise l'intrusion des eaux salées pendant la période des hautes marées. À cela s'ajoute le défaut d'entretien des digues de protection des rizières et des ouvrages hydroagricoles construits à cet effet.

\subsection{Autres Facteurs de la dynamique des paysages agraires}

Le développement rapide de l'arboriculture d'anacardiers et la pression démographique sont également perçus comme des facteurs déterminants de la dynamique des paysages agraires observée surtout au niveau du plateau (figure 5).

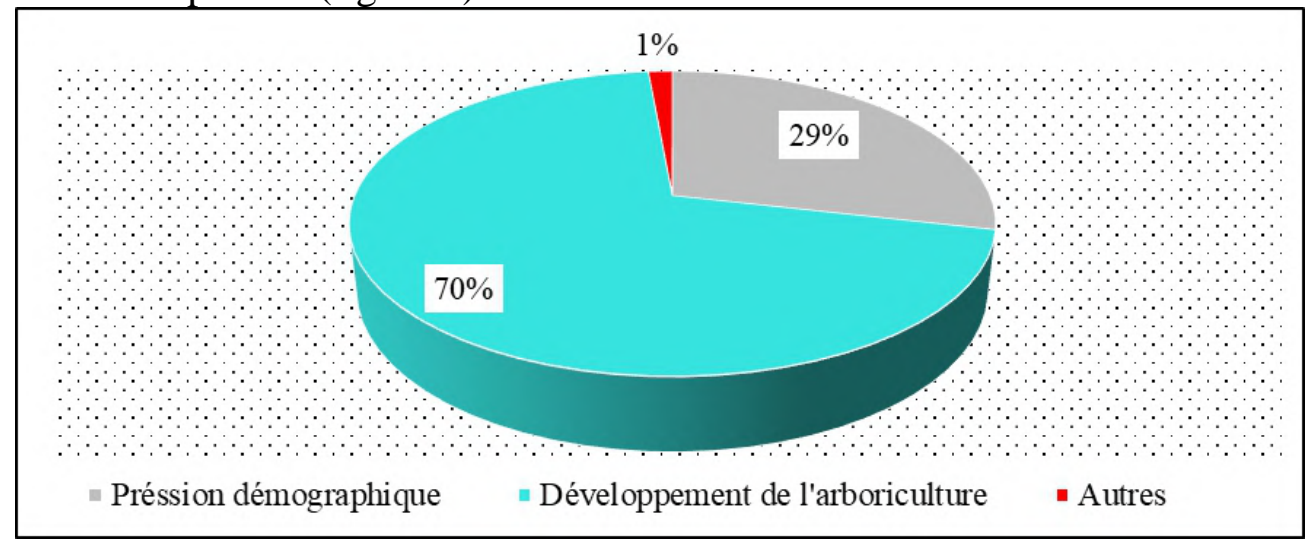

Figure 5 : La perception populaire sur les facteurs anthropiques de l'évolution des paysages agraires de la commune d'Adéane

Environ $70 \%$ des ménages interrogés perçoivent l'arboriculture d'anacardiers comme le principal facteur anthropique de la dynamique des paysages au niveau du plateau. Une bonne partie des terres jadis réservées aux cultures de plateau (arachide, mil, sorgho) est aujourd'hui occupée par les vergers d'anacardiers (photo 1). 


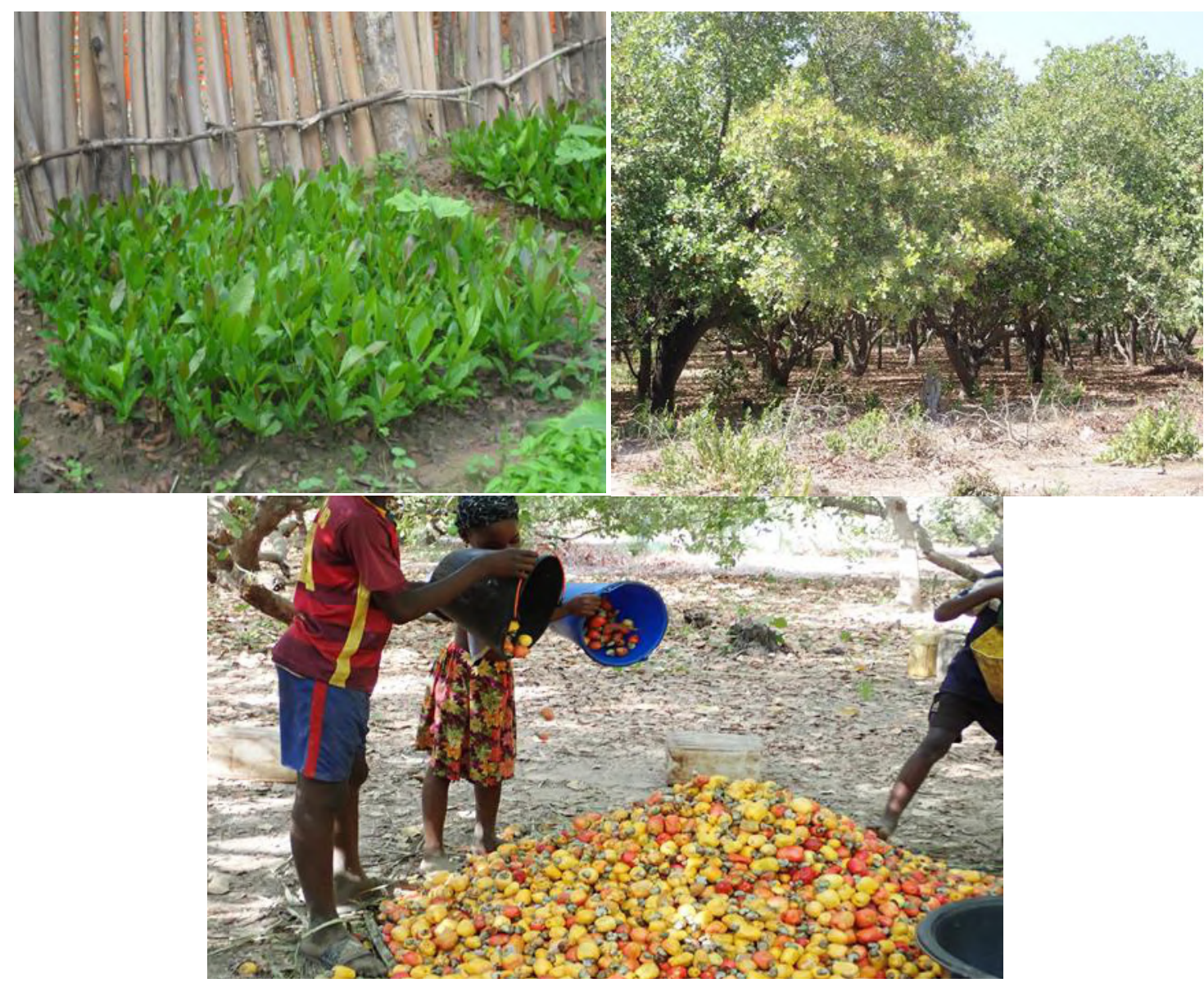

Photo 1 : Développement de l'arboriculture d'anacardiers dans la commune d'Adéane

La première photo montre des pépinières d'anacardiers destinées à la plantation dans les champs pendant l'hivernage et sur la deuxième photo apparaît un champ de culture transformé en verger d'anacardier. Durant les 5 premières de la plantation, les paysans poursuivent les activités agricoles pour permettre le développement rapide des jeunes plantes (Badiane, 2016a). Une fois que les anacardiers colonisent l'espace et commencent à produire, l'activité agricole devient quasi-impossible. La photo 3 illustre l'activité de collecte de la noix, qui se fait par les membres des familles exploitants (femmes, hommes, jeunes garçons et filles).

Outre l'arboriculture d'anacardiers, la pression démographique constitue également un facteur expliquant la dynamique constatée au niveau des zones de cultures du plateau. Avec une population totale estimée à 13114 en 2002 et 17580 habitants en 2013 (ANSD, 2017), la Commune d'Adéane connait une importante croissance démographique au cours des dernières décennies. Cette augmentation de la population a eu des conséquences sur les paysages agraires de la Commune, notamment en termes de demande 
d'espaces pour l'habitat, renforçant ainsi la pression sur le foncier. Cette pression est surtout liée au développement rapide de l'arboriculture d'anacardiers et à l'extension des constructions sur les zones de cultures de plateau. La forte pression exercée par la population de la Commune d'Adéane sur la forêt claire pour la conquête de nouvelles terres de cultures et surtout pour la plantation des anacardiers constitue un élément majeur de la dynamique des paysages agraires de la commune d'Adéane. Cette pression est principalement liée à la flambée du prix du kilogramme de la noix brute sur le marché. L'internationalisation du commerce de la noix avec l'implication des acteurs étrangers (Indiens surtout) à une forte influence sur la variation du prix (Audouin \& Gonin, 2014 ; Sène, 2016 ; Sinan \& Abou, 2016). Le prix du kilogramme bord champ est passé de 35 FCFA en $1992^{4}$ à 750 FCF en $2016^{5}$ (Sène, 2016). Les entretiens réalisés sur le terrain avec les acteurs de la filière anacarde, montre que lors de la campagne 2017 le prix du kilogramme de la noix est vendu entre 1000 et 1200 FCFA bord champ. Ce prix à ensuite connu une légère chute en 2018 (750 FCFA/ kg), puis une importante baisse en 2019 (450 FCFA $/ \mathrm{kg}$ ).

\subsection{Conséquences de la dynamique des paysages agraires sur les activités agricoles}

La dynamique des paysages agraires observée dans la commune d'Adéane, notamment à travers le recul des espaces rizicoles et des zones de cultures de plateau a eu des répercussions sur les activités agricoles de la commune. Aujourd'hui, on assiste à un recul de certaines cultures de plateau à l'image du mil, sorgho et arachide au profit de l'arboriculture d'anacardier. Environ $69 \%$ des ménages interrogés ont affirmé avoir abandonné ces cultures (Badiane, 2016a). La technique de jachère qui permettait de renforcer la fertilité des terres est en voie de disparition. Environ $42 \%$ des ménages interrogés ont abandonné cette pratique (Badiane, 2016a) en raison de l'insuffisance des espaces de cultures liée à l'expansion démographique et au développement de l'arboriculture d'anacardiers sur le plateau.

La conséquence de la dynamique des paysages agraires au niveau des zones rizicoles est l'abandon progressif de l'activité rizicole au profit de l'arboriculture d'anacardiers. Le temps de travail, jadis, consacré à l'entretien des rizières (réhabilitation des digues et de diguettes de protection des parcelles rizicoles, et des ouvrages hydroagricoles) est de plus en plus utilisé pour la conquête de nouvelles terres du plateau destinées aux plantations d'anacardiers. Malgré l'intervention de l'ANCAR et du Projet Natal Mbaye, pour sauver l'activité rizicole dans la commune d'Adéane (distribution des

${ }^{4}$ Avant l'arrivée des Indiens

${ }^{5}$ Forte influence des Indiens (arrivés en 1995 dans le marché de la commercialisation de la noix au Sénégal) sur l'augmentation des prix 
semences de riz certifiées et à cycle plus court, accompagnement des riziculteurs), la production ne cesse de baisser et la population se tourne dans la production de l'anacarde.

\section{Discussions}

Les résultats cartographiques montrent que les paysages agraires de la commune d'Adéane ont connu une importante dynamique entre 1968, 2004 et 2016. Cette dynamique est généralement marquée, d'une part, par la régression des superficies de rizières exploitées au profit des rizières abandonnées et des surfaces de tannes et d'autre part, par la naissance et la progression des vergers d'anacardiers et des zones d'habitation sur les zones de cultures de plateau et sur la forêt claire. Par conséquent, les activités agricoles, notamment les cultures céréalières (riz, mil, maïs) et arachidières, connaissent une importante baisse ces dernières années au profit de l'arboriculture d'anacardiers (Badiane, 2016a).

En effet, le développement de la filière anacarde au cours de ces dernières années s'est accompagné par de profondes recompositions sociospatiales (Audouin \& Gonin, 2014). L'étude réalisée par le Groupe de recherche et de réalisations pour le développement rural (GRDR) à l'échelle de la Casamance et de la Guinée-Bissau, montre que les zones de cultures pluviales et les réserves (jachère, forêt et palmeraie) régressent au profit des vergers et du bâti (GRDR et al., 2017). Cette étude a également montré que la tendance est encore plus marquée dans les localités de la Guinée-Bissau, où les vergers occupent généralement 20 à $30 \%$ des superficies exondées. Dans ce même sillage, Diop (2017) a montré que les superficies de la classe rizière exploitée ont régressé de 27,79\% entre 1972 et 2016. Ces différentes études corroborent nos résultats qui montrent une importante régression des rizières exploitées entre 1968 et $2016(45,45 \%$ pour le terroir d'Adéane et 55,65\% pour le terroir d'Agnack-Petit) et une forte augmentation des superficies de vergers d'anacardiers.

Par ailleurs, une particularité se présente sur les principaux facteurs de la dynamique des paysages agraires observée surtout au niveau des rizières dans la Commune d'Adéane. La plupart des études réalisées jusque-là en Basse-Casamance ont montré que la dégradation et l'abandon de certaines rizières ainsi que le recul de l'activité rizicole sont principalement liés à la salinisation et à l'acidification des terres (Barry et al., 1988 ; Diouf, 2013 ; Marius \& Lucas, 1982 ; Montoroi, 1994). Toutefois, nos résultats ont montré qu'en plus des facteurs physico-chimiques (salinisation et acidification), le développement de l'arboriculture d'anacardiers apparaît comme un facteur expliquant le recul de l'activité rizicole dans la commune d'Adéane.

En effet, la population de la Commune d'Adéane se tourne davantage vers la production de l'anacarde, qui apparait aujourd'hui comme la principale 
activité génératrice de revenus dans la commune. Le rythme d'évolution des plantations d'anacardiers augmente d'année en année (Badiane, 2016a) et parallèlement, on constate un recul progressif des champs de cultures. Le facteur prix est mis en avant dans l'explication de la convergence des paysans de la commune d'Adéane dans le secteur de l'arboriculture d'anacardier. L'étude réalisée en Côte d'Ivoire par Sinan et Abou (2016) a montré que le prix bord-champs de la noix de cajou a évolué de 125 FCFA en 2010 à 350 FCFA en 2016. Dans cette même logique, Sene (2016) a montré que dans la région de Ziguinchor, le prix de la noix a évolué de 35 FCFA en 1992 à 750 FCF en 2016. Cette évolution du prix s'est poursuivie jusqu'en 2017 (entre 1000 et $1200 \mathrm{FCFA} / \mathrm{kg}$ ) avant de connaitre une baisse continue pendant les campagnes 2018 (750 FCFA/kg) et 2019 (450 FCFA/kg) .

\section{Conclusion}

Il est important de retenir que cette recherche renseigne sur la dynamique des paysages agraires, les principaux facteurs liés à cette dynamique ainsi que ses conséquences sur les activités agricoles de la commune d'Adéane. La cartographie diachronique de l'occupation des sols a permis de voir, d'une part, un recul des rizières exploitées avec un taux de régression de 45,45\% pour Adéane et 56,64 \% pour Agnack-petit entre 1968 et 2016 et, d'autre part, une rapide progression des vergers d'anacardiers (228,46\% pour Adéane et 519,28\% pour Agnack-petit) au détriment des champs de cultures de plateau et de la forêt claire. Ce travail de recherche a permis également d'apprécier la situation actuelle de la riziculture, qui est en recul en raison de la dégradation des conditions agro-pédologiques (salinisation et acidification). Le recul des activités agricoles, notamment rizicoles, est surtout amplifié par le développement rapide de l'arboriculture d'anacardiers ces dernières années. Celle-ci attire une masse importante de paysans de la commune d'Adéane, en raison de sa valeur économique. Toutefois, la forte affluence de la population vers la monoculture de l'anacarde suscite un ensemble d'interrogations qui méritent, en termes de perspectives, d'être analysées. Il s'agit notamment de la question de l'avenir de la riziculture, dans ce contexte des changements globaux, et de l'impact écologique du développement rapide de la monoculture d'anacardiers et sa durabilité dans la commune d'Adéane. De surcroît, une réflexion approfondie sur les enjeux fonciers liés au développement rapide de l'arboriculture d'anacardiers mérite d'être conduite.

\footnotetext{
${ }^{6}$ Entretiens réalisés avec les producteurs et le secrétaire général de la chambre de commerce de Ziguinchor
} 


\section{References:}

1. ANSD. (2017). Recensement Général de la Population et de l'Habitat, de l'Agriculture et de l'Elevage (RGPHAE 2013) (p. 83) [Rapport Régional définitif]. Ziguinchor.

2. Audouin, S., \& Gonin, A. (2014). L'anacarde: produit de la globalisation, moteur de la territorialisation, l'exemple du Sud du Burkina Faso. EchoGéo, (29). https://doi.org/10.4000/echogeo.13926

3. Badiane, A. (2016a). Caractérisation et dynamique des systèmes de production agricole de la commune d'Adéane (Basse Casamance) (Mémoire de master). Université Assane SECK de Ziguinchor.

4. Badiane, L. M. (2016b). Gestion hydrologique et spatiale d'un hydrosysteme aménagé: le marigot de Bignona en Basse-Casamance (Thèse de doctorat). Université Gaston Berger de Saint-Louis (Sénégal).

5. Barry, B., Boivin, P., Brunet, D., Montoroi, J.-P., Mougenot, B., Touma, J., ... Eau et Développement : Journées de 1'Eau au Sénégal, 2., Dakar (SEN), 1988/12/08-10. (1988). Evolution des stratégies d'aménagement hydro-agricole des sols salés en Basse Casamance. Deuxièmes journées de l'eau au Sénégal: eau et développement. Présenté à Dakar. Consulté à l'adresse http://www.documentation.ird.fr/hor/fdi:27577

6. Centre de Suivi Ecologique. (2010). Rapport sur l'état de l'environnement au Sénégal (p. 268). Dakar: Ministère de l'Evironnement et de la Protection de la Nature.

7. Communauté Rurale d'Adéane. (2008). Plan local de Développpement de la communauté rurale d'Adéane (p. 63). Adéane.

8. Cormier-Salem, M.-C. (1992). Gestion et évolution des espaces aquatiques: la Casamance (Editions ORSTOM). Paris.

9. Descroix, L., Diongue Niang, A., Panthou, G., Bodian, A., Sane, Y., Dacosta, H., Malam Abdou, M., Vandervaere, J-P., Quantin, G. (2016). Évolution récente de la pluviométrie en Afrique de l'ouest à travers deux régions: la Sénégambie et le Bassin du Niger Moyen. Climatologie, (Volume 12). https://doi.org/10.4267/climatologie.1105

10. Diop, A. (2017). L'évolution de l'occupation des sols et de la maind'euvre rizicole dans le Bandial en Basse Casamance (Mémoire de master). Université Assane SECK de Ziguinchor.

11. Diouf, E. (2013). Ouvrages hydrauliques et modèles de gestion de l'eau dans le bassin du fleuve Casamance (Thèse en cotutelle internationale). Université Gaston Berger de Saint-Louis (Sénégal) et Université Paris Ouest-Nanterre la Défense (France).

12. GRDR. (2017). Un littoral en mouvement Ziguinchor - Sedhiou - Oio - Cacheu: diversité, dynamiques et mutations des territoires frontaliers 
du sud-ouest du Sénégal et du nord-ouest de la Guinée-Bissau. Montreuil: GRDR.

13. Marius, C., \& Lucas, J. (1982). Evolution géochimique et exemple d'aménagement des mangroves au Sénégal (Casamance). Oceanologica Acta, (No spécial), pp 151-160. (Centre IRD de Bondy).

14. Montoroi, J.-P. (1994). Dynamique de l'eau et géochimie des sels d'un bassin versant aménagé de Basse-Casamance (Sénégal): conséquences sur la gestion durable de l'écosystème de mangrove en période de sécheresse (PhD Thesis). Université Henri Poincaré-Nancy 1.

15. Ndiaye, S., Charahabil, M. M., Ndiaye, O., et Diatta, M. (2017). Influence de la flore ligneuse associée dans la production des parcs à Anacardium occidentale L. dans la communauté rurale de Djibanar (Casamance/Sénégal). International Journal of Biological and Chemical Sciences, 11(2), pp 585-596.

16. Sané, T. (2017). Vulnérabilité et adaptabilité des systèmes agraires a la variabilité climatique et aux changements sociaux en BasseCasamance (Thèse de Doctorat en co-tutelle). Université Cheikh Anta Diop de Dakar et Université Sorbonne Paris Cité Préparée à l'Université Paris Diderot-Paris 7.

17. Sène, A. M. (2016). Agrobusiness de l'anacarde en Casamance: atouts, contraintes et perspectives d'industrialisation. In Nourrir l'Afrique: vers une agro-industrialisation pour une croissance inclusive, pp 19.

18. Sinan, A., et Abou, N. K. (2016). Impacts Socio-Economiques De La Culture De L'anacarde Dans La Sous-Prefecture D'odienne (Côte d'Ivoire). European Scientific Journal, ESJ, 12(32), 369. https://doi.org/10.19044/esj.2016.v12n32p369

19. Thior, M., Sy, O., Sané, T., Mballo, I., Badiane, A., et Descroix, L. (2019). Contraintes à la production rizicole et reconversion socioéconomique dans la commune de Diembéring (Sénégal). Revue Ivoirienne de Géographie des Savanes, (6), pp 13. 